ducing advanced signs of irrefutable malignant disease. The patient made it perfectly clear to each candidate that the ulcer had been stationary for over six months; in fact, it appeared to be getting smaller lately. It was unlikely, although I agree that it is possible, that an epithelioma of the cheek should, at the same time, be associated with a like growth in the tongue.

The subsequent history of this case has shown that on appropriate anti-syphilitic treatment and hygiene of the mouth both ulcers are now rapidly healing, and the patient is very much improved in every respect.

\title{
A CASE OF CYSTIC SWELLING IN THE GROIN.
}

\section{By A. J. COKKINIS, M.B.LOND., F.R.c.s.}

THIS case, demonstrated in the F.R.C.S. course of the Fellowship of Medicine, presents an interesting problem in diagnosis. The patient, a man in the early twenties, complained of an intermittent limping which appeared six months History. previously. Walking was quite painless and the limping occurred at intervals for no apparent reason. Three weeks before the demonstration a swelling appeared in the right groin and has slowly increased in size. The condition has been painless throughout. There is no history of injury.

On examination, there is a prominent, fluctuating swelling above the outer half of Poupart's ligament, oval in shape, and about 3 by 2 in. in area; it is quite painless, cold and non-tender, and the skin over it is unaltered. There is a

Physical Signs.

doubtful impulse on coughing. The swelling is not translucent and it appears to lie deeply to the abdominal wall. The spine, hips and sacro-" iliac joints seem quite normal, and radiograms of all these regions show no lesion whatever. There is, perhaps, slight limitation of hyper-extension of the right hip, but all other movements are free and painless. The patient appears quite healthy and welldeveloped. Rectal examination is negative.

The following were the diagnoses suggested by various post-graduates attending the course : (1) Femoral hernia ; (2) interstitial inguinal hernia ; (3) lipoma ; (4) an abscess coming from the appendix or cæcum; (5) a psoas abscess from Discussion. a spinal caries; (6) an abscess complicating tuberculous hip; (7) an abscess from sacro-iliac tubercle; (8) an enlarged psoas bursa ; (9) a cold abscess following tuberculosis of the iliac lymph glands.

A critical analysis of these diagnoses is instructive.

This was suggested by two post-graduates, but is wrong, for the very

(1) Femoral simple reason that the swelling nowhere extended below Poupart's Hernia. ligament.

A very popular diagnosis and one which should be suggested; but acquired

(2) Interstitial Inguinal Hernia. interstitial hernia is nearly always traumatic in origin. Moreover, this swelling is very definitely cystic, the impulse on coughing is extremely doubtful, and the pre-existing limp does not fit into this diagnosis.

This also proved a popular diagnosis and is one which would be expected by

(3) Lipoma.

most examiners. The extreme fluctuation, the absence of lobulation or of a definite edge, and again the limping, however, almost 
(4) Appendix Abscess.

(5), (6) and (7) Absces:

Complicating Disease of the Spine, Hip or Sacro-iliac Joints.

(8) Enlarged Psoas Bursa.

A bad diagnosis, because of the absence of suggestive history and inflammatory signs.

One or other of these was suggested by practically every post-graduate. The absence of any symptom or sign of disease in these regions and the normal radiograms must be accepted as conclusive evidence against such a diagnosis.

A very reasonable suggestion made by one candidate only. One would expect the bursal swelling to be more medial in position, but the possibility of an abnormally extensive bursal sac should have been borne in mind. This is the diagnosis which most nearly agrees with the clinical picture, but was only made by three post-graduates. Tuberculous adenitis of the iliac glands is a wellknown condition, and the close proximity of the glands to the psoas (9) Tuberculous muscle is an accepted cause of psoitis; this gives a reasonable explanIliac Glands.

ation of the intermittent limp which preceded the swelling by more than five months. The glands would eventually soften and break down, and the resulting abscess would track outwards under the iliac fascia and point in the position of the swelling. There it would present all the features of a cold abscess-precisely the picture given by the case under discussion.

It is interesting to add that the swelling was aspirated a day or two after the case was shown, and the fluid withdrawn proved to be a typical tuberculous pus.

\section{A TUMOUR OF VOLUNTARY MUSCLE.}

\section{BY HAMILTON BAILEY, F.R.C.S.}

A WOMAn aged 32 was presented with a swelling on the inner side of the thigh. She stated that she had noticed a lump for the first time three months

History. previously; it was then the size of a walnut. Growth had been slow for two and a half months, after which enlargement had been more rapid. Pain was a feature during the past three weeks only.

The patient was a healthy-looking woman. There was a swelling the size of an orange on the inner side of the lower third of the right thigh. The overlying skin presented a normal appearance, but it was slightly tethered over the

Clinical Signs. maximal convexity of the lump. The swelling was hard, obviously solid, and regularly irregular. It could be freely moved from side to side but not from above downwards. On abducting the thigh or asking the patient to voluntarily contract the muscles of the inner side of the thigh it was at once apparent that there was a cessation of lateral mobility.

Almost without exception post-graduates correctly elicited the physical signs, although a proportion failed to demonstrate the last mentiomed sign, which typifies a swelling connected with a muscle. In this case the group of muscles with which the swelling was associated were the hamstrings, and because the swelling was situated rather at the back of the group and because it was connected with a fleshy muscle as opposed to a tendon, it seemed probable that the actual muscle connected with it was the semi-membranosus. When asked the nature of the swelling very few members of the class would venture an opinion, and "it might be a lipoma" seemed the favourite 